\title{
Vascular Changes in Peripheral Corneal Destructive Disease
}

\author{
P. G. WATSON
}

Cambridge

\begin{abstract}
Summary
A clinical classification of peripheral corneal destructive disease is presented. These syndromes all start within $2 \mathbf{~} \mathbf{m m}$ of the limbus and are accompanied by varying degrees of vaso-occlusion of the adjacent limbal vascular networks. Close observation of the configuration, integrity and pattern of the limbal vessels can indicate whether the disease is active, quiescent or brought under control with treatment.
\end{abstract}

Loss of corneal tissue occurs in many conditions and may result from an active destructive process directly associated with bacterial or viral invasion, or from the local immune response to these organisms or as a part of a systemic immunologically induced disorder.

Characteristically these lesions, which are associated with disorders of the adjacent vasculature, occur at the periphery of the cornea and although they may spread to the centre of the cornea they rarely do so as a primary event. It is the purpose of this paper to identify the clinical syndromes which give rise to peripheral corneal destruction and to note the vascular changes which are detectable clinically in these conditions. ${ }^{1}$

\section{Clinical Syndromes}

Clinically five types of peripheral distructive corneal disease are recognisable. They are accompanied by varying amounts of inflammation, which may be either confined to the limbus itself or to the adjacent sclera.

\section{Peripheral Corneal Thinning (Contact Lens Cornea)}

Of all the conditions in which corneal tissue is lost peripheral corneal thinning is the most benign. These patients, almost all of whom have longstanding rheumatoid arthritis, show gradual resorbtion of the corneal tissue in such a way that the peripheral cornea is very thin but the central cornea remains of normal thickness and transparency looking as if a contact lens has been placed on the cornea. There is little or no inflammation at the limbus. Careful inspection shows the limbal arcades to be broken and irregular and in some instances the limbal blood vessels invade the cornea either in the mid stroma or as a pseudo pterygium, or both (Fig.1).

\section{Terriens Disease}

In Terrien's disease there is a sharp demarcation of the thinning of the cornea about 2-3 mm from the limbus. The cornea central to the lesion is infiltrated and the cornea may become thin, either from superficial or from the deep surface. The thinning may be extreme but the deep peripheral cornea is always vascularised (Fig. 2). However at no stage in the disease do these vessels appear to be active in that they form new vessel loops gradually which do not leak. Serum residues build up at the limbus but are sometimes almost where new vessels have sutured corneal tissue. 


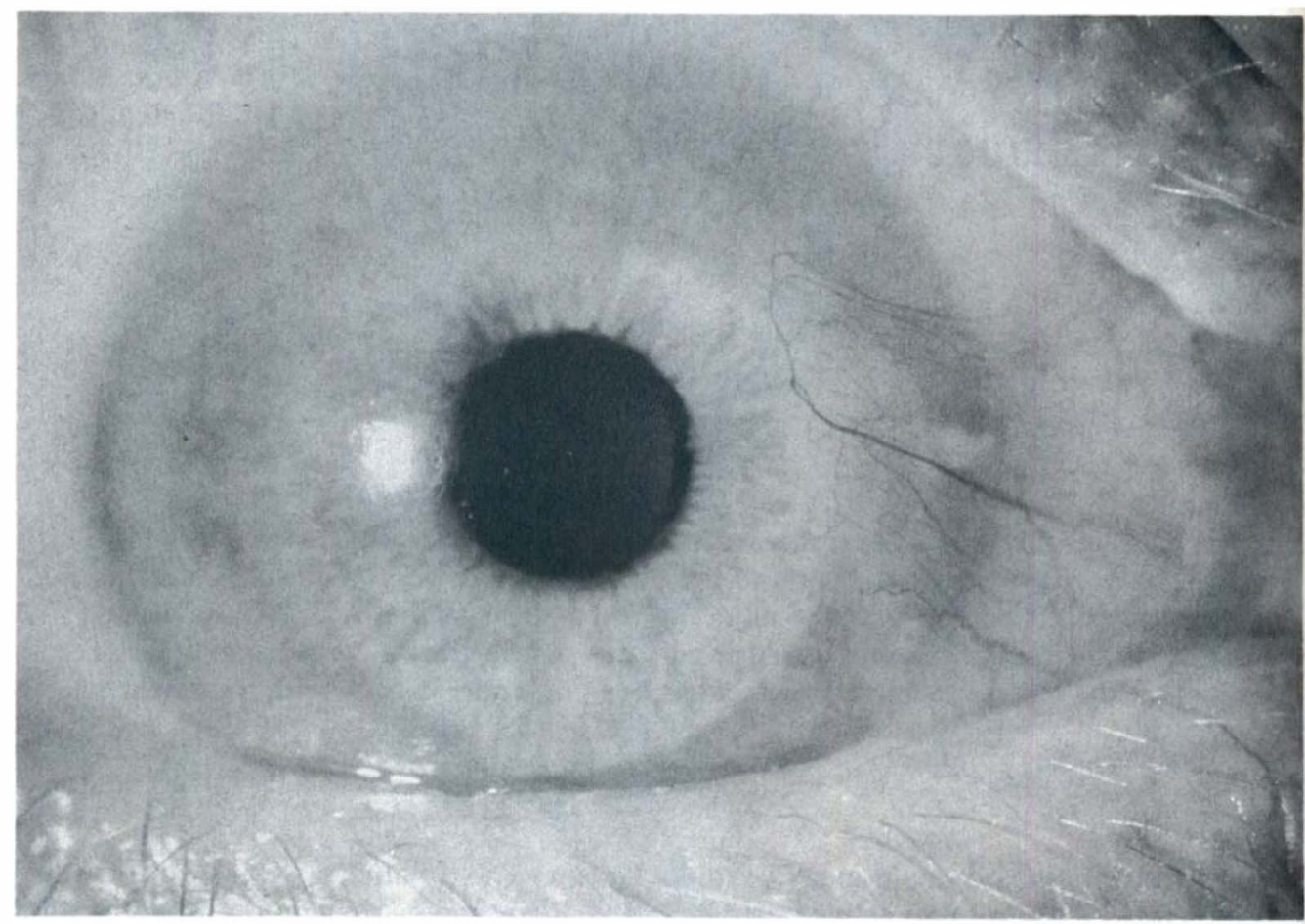

Fig. 1. Contact lens corneas in a patient with long standing rheumatoid arthritis. He had no complaints about his vision at this stage. The peripheral cornea is thin but transparent. There is no limbal or scleral inflammation and there are no corneal infiltrates but the limbal arcades are broken and vessels are invading the cornea both from the conjunctival (as a pseudo-pterygium) and the mid stromal limbal vessels.

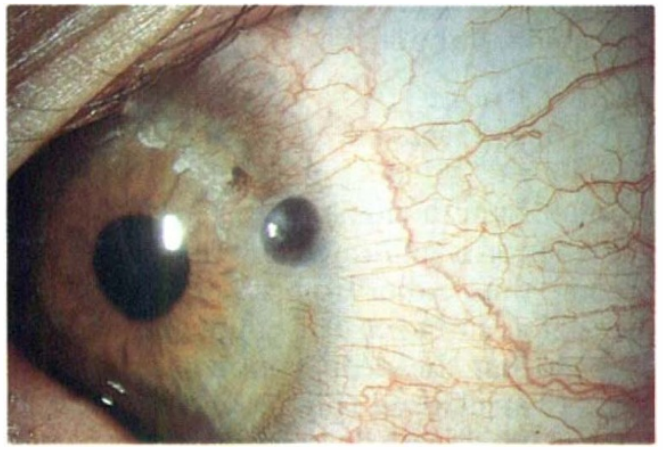

Fig. 2. Terriens disease in a 40 year old man. The peripheral cornea has become thin and in one phase the iris has permeated into a deep defect. All the vascular loops from the limbus are closed; the vascular invasion of the cornea is limited to the area of affected cornea which is infiltrated with lipid central to the thinned areas.

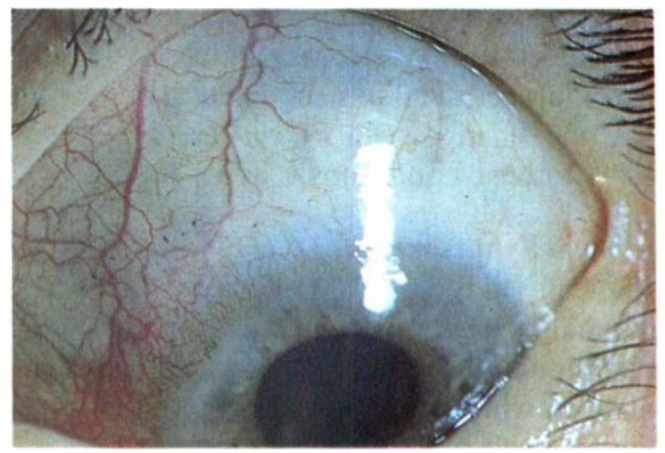

Fig. 3a. Early peripheral corneal melting at 10 o'clock. There is very little limbal inflammation but the cornea is infiltrated and central to the gutter. 


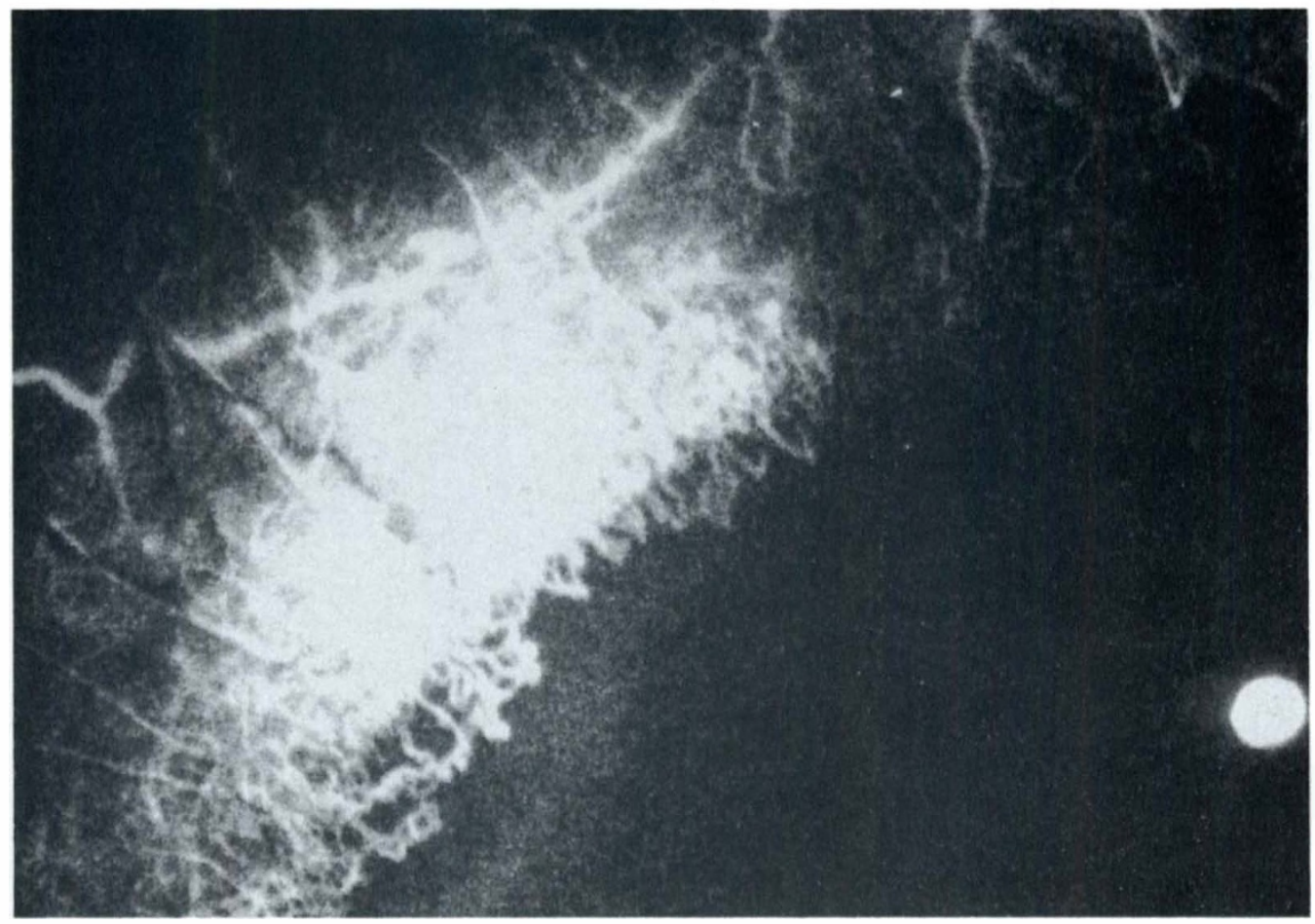

Fig. 3b. Anterior segment fluorescein angiography of the same lesion shows intense vasolidation of the vessels in the region of the gutter. At the advancing superior edge the limbal arcades have extended into the cornea with straight unconnected vessels. When the gutter has become established those loops have joined. All the vessels leak fluorescence at their tips.

\section{Peripheral Corneal Melting}

Peripheral corneal melting may arise in an area of already thinned peripheral cornea of the contact lens type and without severe inflammation. Vessels at the limbus extend into the cornea around the area of tissue loss. These vessels are straight and have a rapid rate of flow (Figs. 3 and 4). More commonly, there is intense inflammation at the limbus which may or may not involve the adjacent sclera. The cornea becomes grey, swollen and infiltrated. The initial lesion tends to break down and extend peripherally but rarely centrally (Fig. 4). Occasionally peripheral corneal melting occurs without any associated infiltration. The gutter thus formed usually has a sharp, well defined border both on it's limbal and corneal edges, although the margin may well be undermined (Fig. 5). During the active phase of the disease the vessels of the limbus invade the cornea, are straight and leak at their tips (Figs. 6a,b).

\section{Acute Stromal Keratitis or Keratolysis}

Destructive stromal keratitis is always associated with intense inflammation of the limbus or the adjacent sclera. Initially the cornea becomes infiltrated and thickened close to the

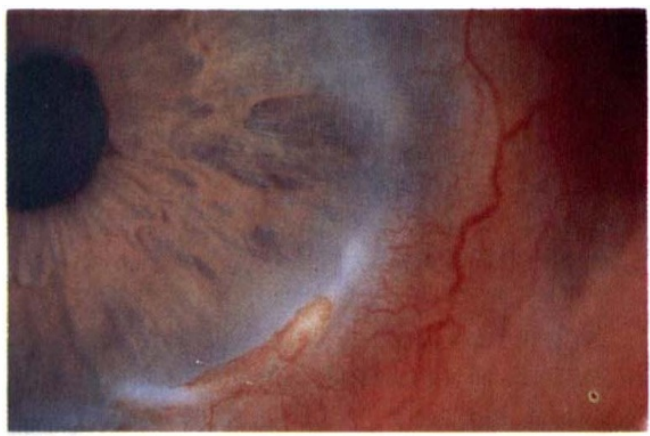

Fig. 4. Limbal infiltration at the commencement of a limbal gutter. The cornea is invaded by a rapidly advancing capillary net. The limbal arcades remote from the infiltrated area are disturbed and no longer regular and there is intense inflammation of the adjacent limbus. 


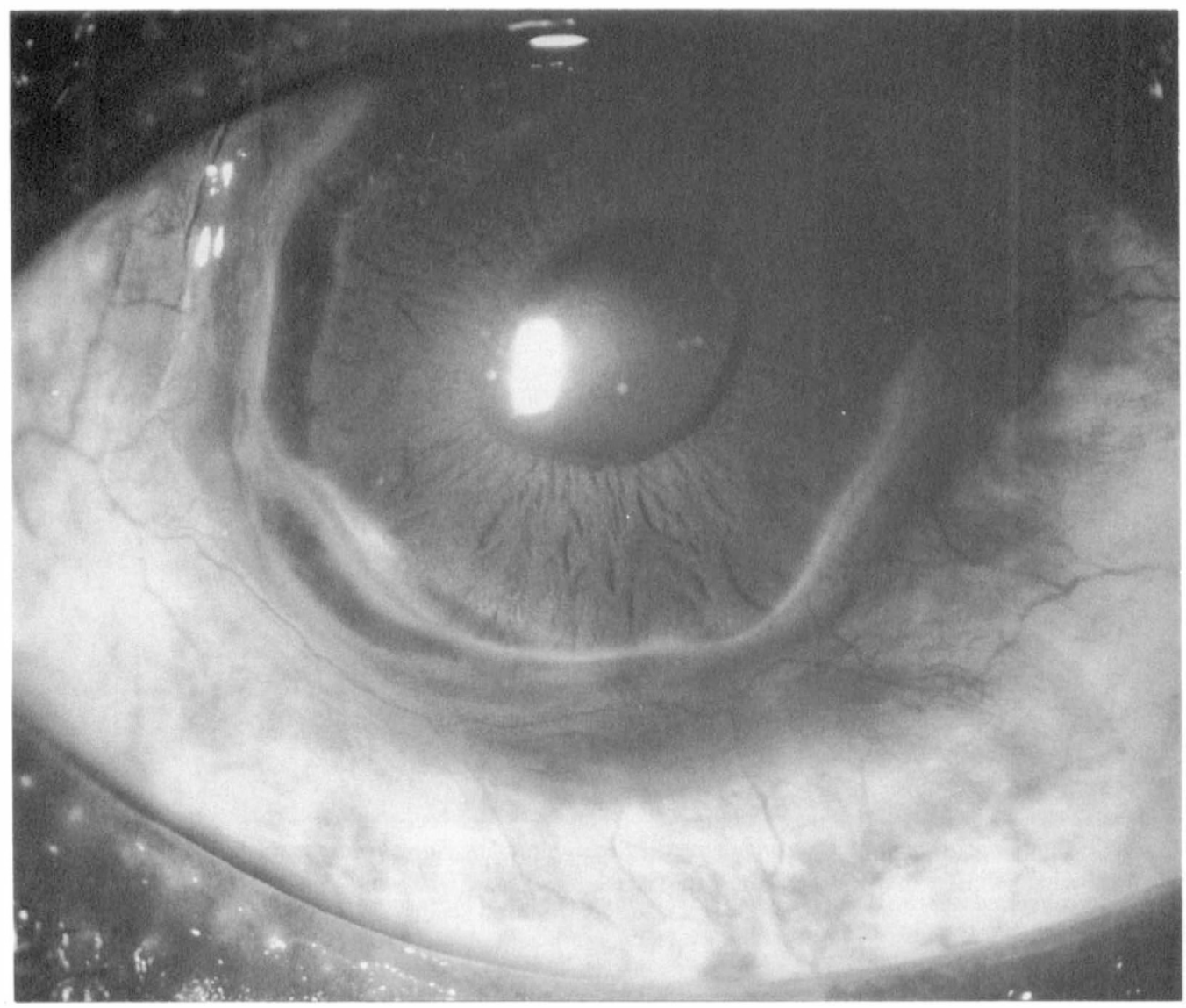

Fig. 5. Extreme thinning of the peripheral cornea without any adjacent inflammation in a patient with long standing rheumatoid arthritis.

limbus and usually within $2 \mathrm{~mm}$ from its edge. These white and grey precipitates coalesce so that large areas of the cornea eventually become involved. There may be some keratic precipitates underneath the area of inflammation. In due course the surface epithelium is eroded and loss of stromal tissue occurs (Fig. 7).

Destructive lesions which transgress the limbus are always associated with a systemic vasculitis (Fig. 8). Before, or simultaneous with the destruction of corneal or scleral tissue the limbal arcades become completely disrupted and vessels invade the cornea at all levels (Fig. 9). There is also non-perfusion of the adjacent episcleral and conjunctival vascular networks.

\section{Mooren's Ulceration}

Mooren's ulceration is extremely difficult to distinguish from any other form of corneal guttering in its initial stages but it does appear to be a specific entity. This extremely painful ulcer starts adjacent to the corneal scleral limbus. There is stromal infiltration and corneal thickening in advance of the ulcer which is always undermined. The ulcer progresses both centrally and peripherally leaving thin vascularised cornea (Figs. 10, 11a,b). The central cornea thickens and becomes opaque, and eventually if it does not perforate becomes scarred and paper thin (Fig. 12). There is no scleral involvement, the inflammatory response is variable and it is not associated with any other systemic disorder. The 


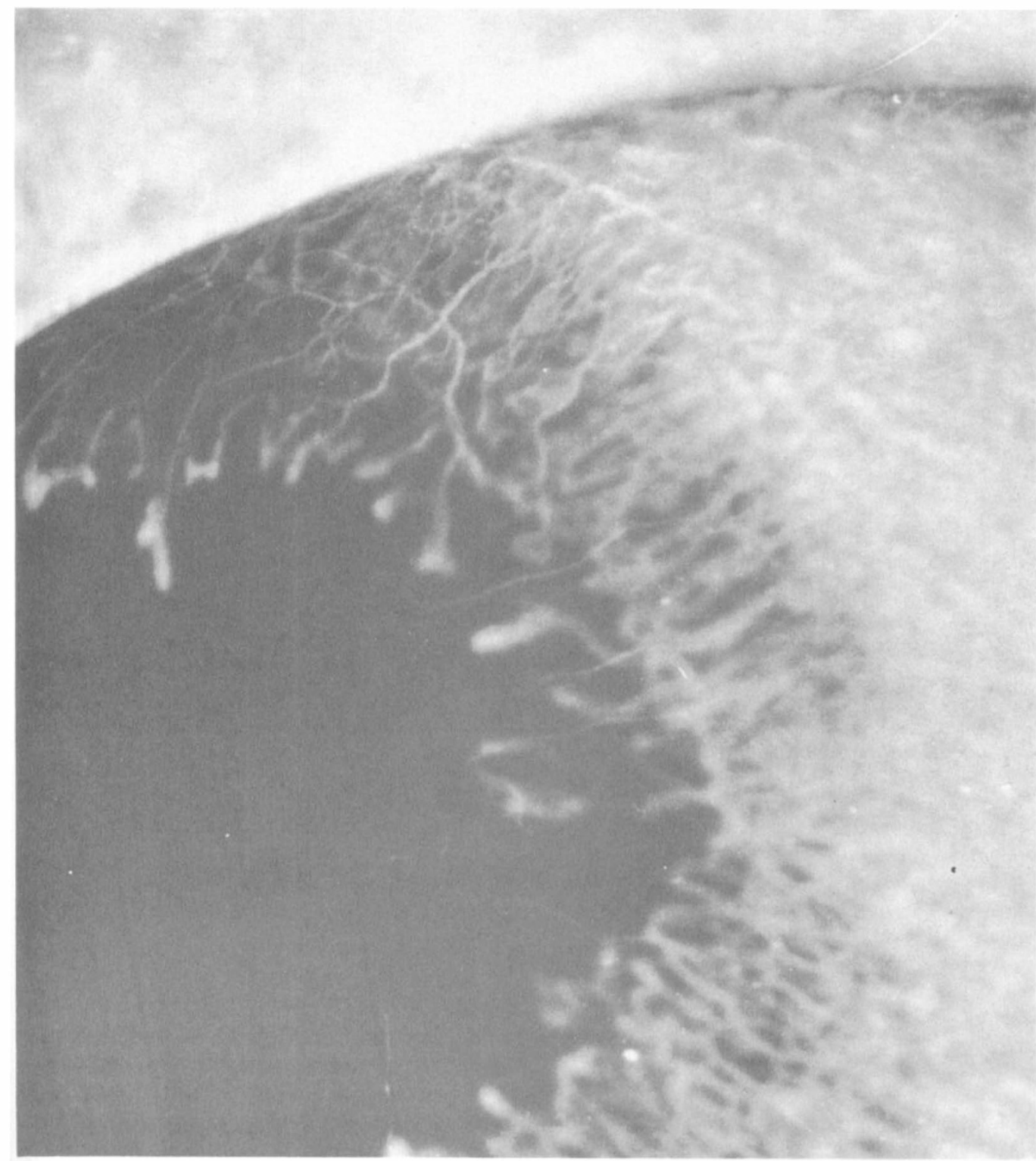

Fig. 6a. Vascular invasion during reactivation of a peripheral corneal guttering. The vascular loops from a previous episode have closed and do not leak, but new vessels have sprouted from these loops to invade previously unaffected cornea. These loops are straight and leak at their tips.

vascular networks at the limbus become disrupted before the appearance of the ulcer at that site. New vessels sprout from the superficial conjunctival capillaries and extend to the advancing edge of the ulcer. In the active stage of the disease these vessels leak at the advancing tips.

Furthermore, the vaso-occlusive changes precede the appearance of destructive corneal changes which once they have occurred are permanent; areas thus affected may therefore be subject to new vessel formation.

Close observation of the limbal capillaries and adjacent episcleral network either clinically, using red freelight, or with anterior segment fluorescein angiography can indicate 


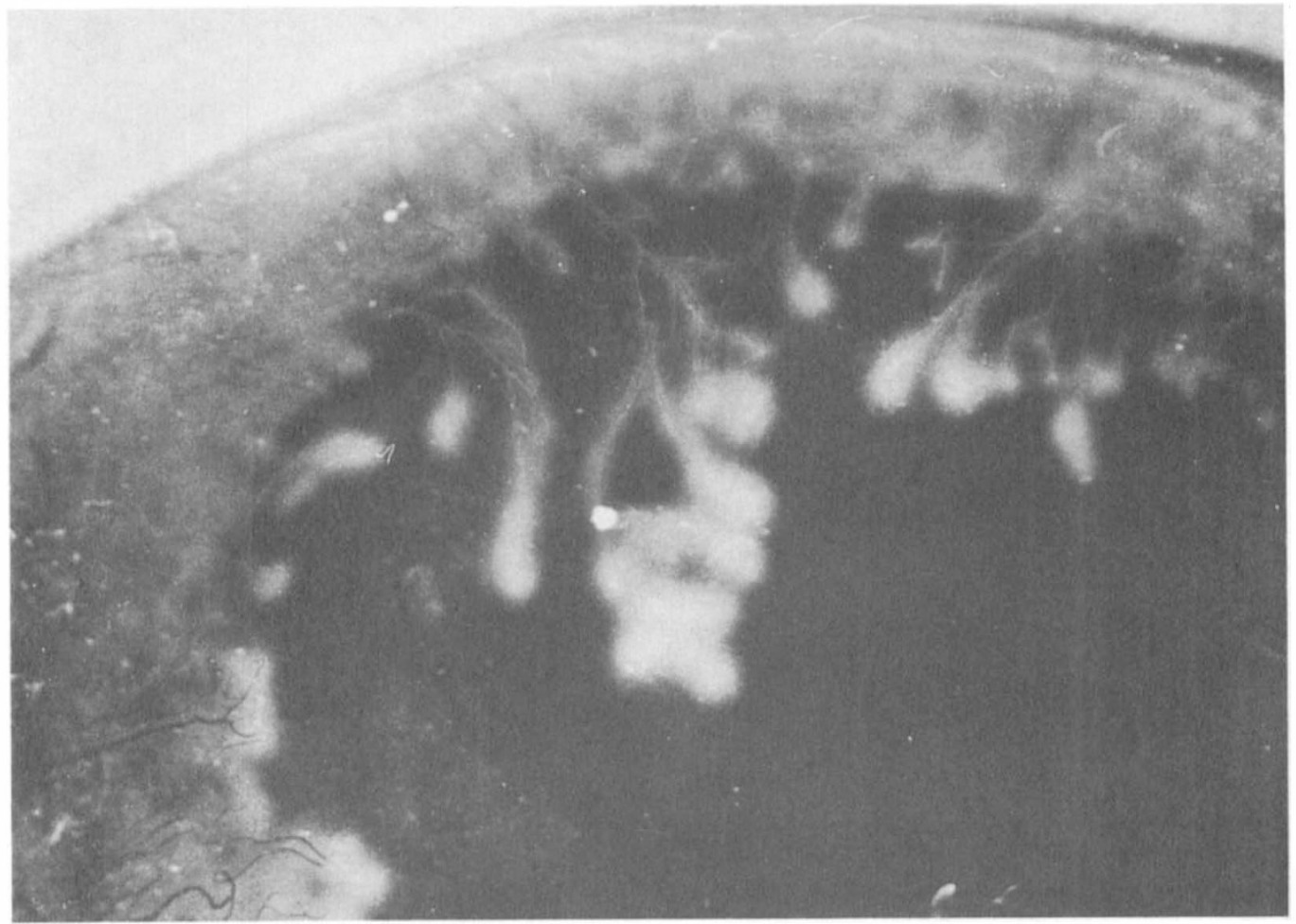

Fig. 6b. Late phase of this angiogram nasally in the same eye as Fig. $6 a$.

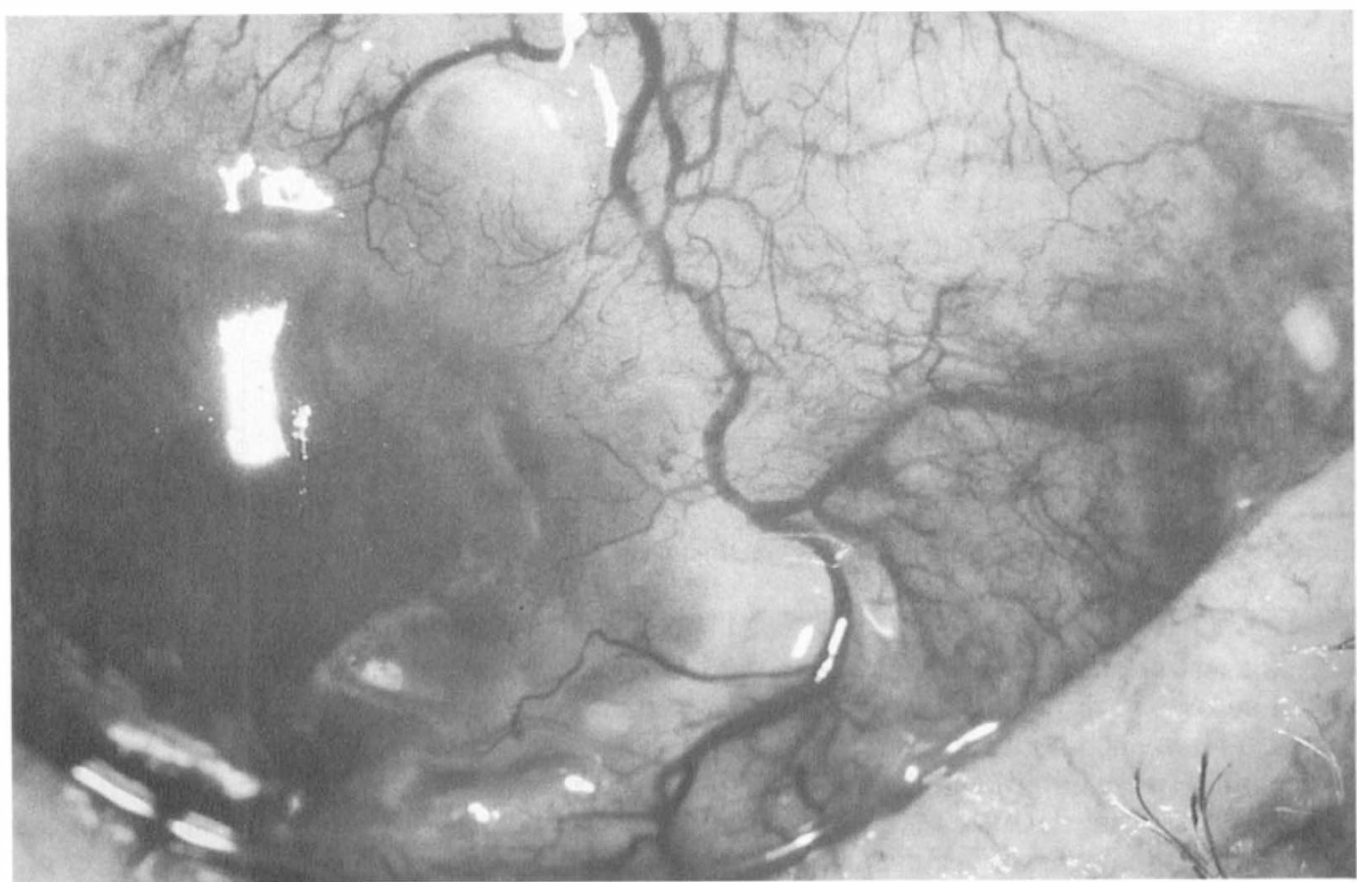

Fig. 7. Acute stromal keratitis. The limbal architecture has completely disappeared and is replaced by inflammed tortuous abnormal vessels between which the cornea has been destroyed. 


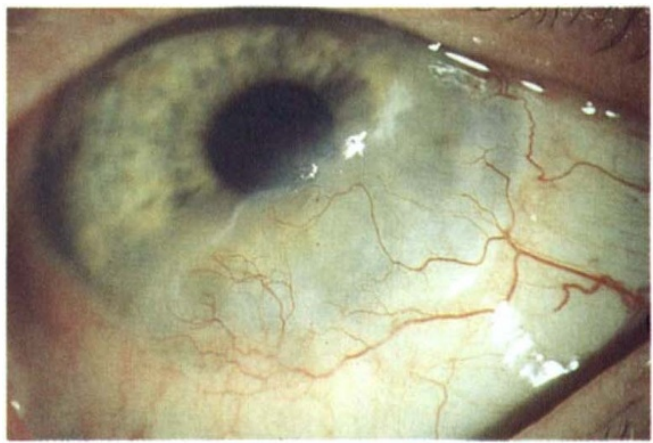

Fig. 8. A similar patient to that shown in Fig. 7 after treatment with pulsed immunosuppressive therapy. Serum residues remain at the edge of the ulcerated cornea. The vessels run to the edge of this ulcer but have joined together indicating that there is no further activity in the disease process. The normal limbus has completely disappeared.

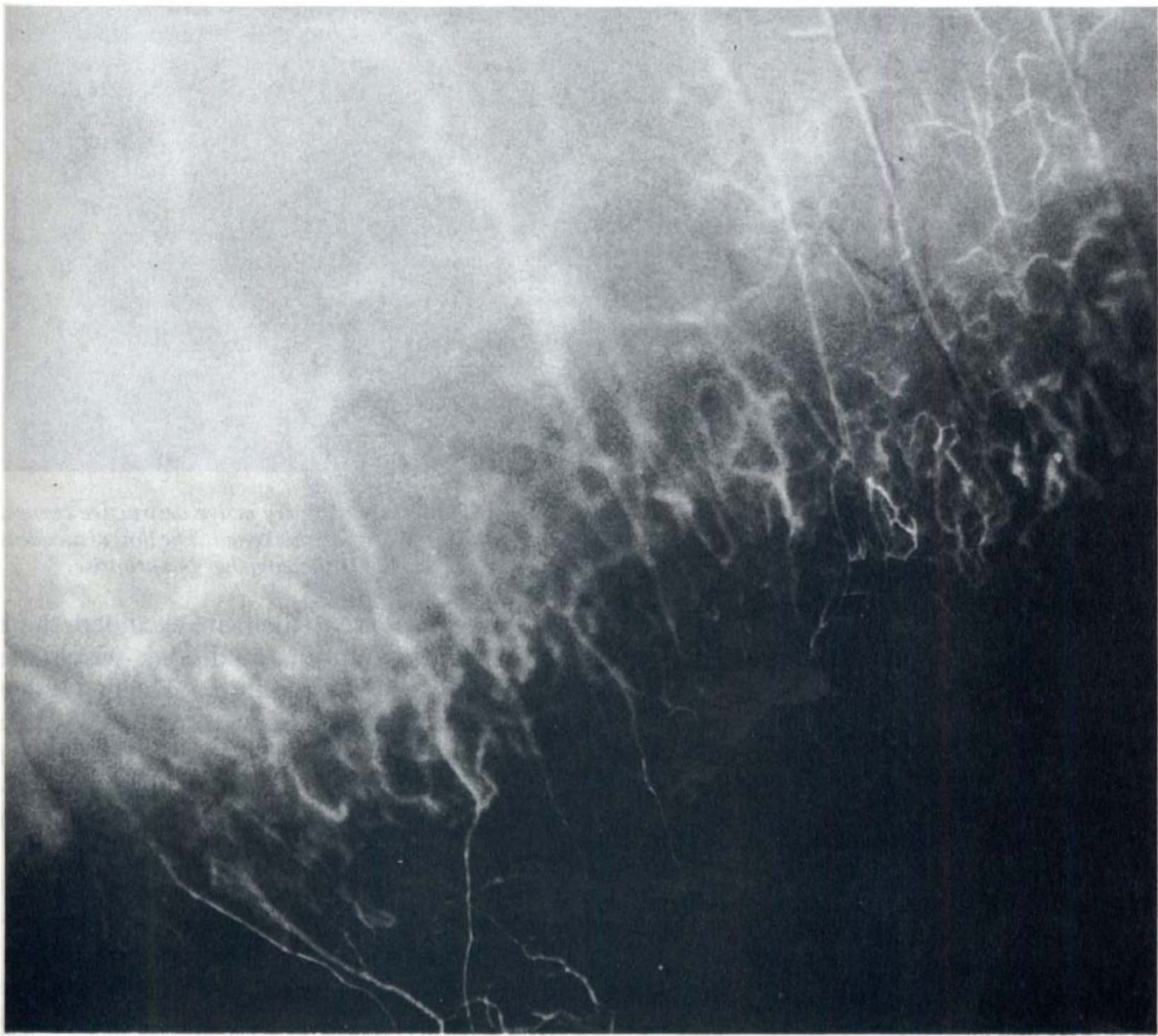

Fig. 9. Vascular loops after treatment of acute stromal keratolysis. The vessels are looped at their tips (whatever layer they are derived from) and do not leak fluorescenin. The normal limbal architectures is disrupted. 


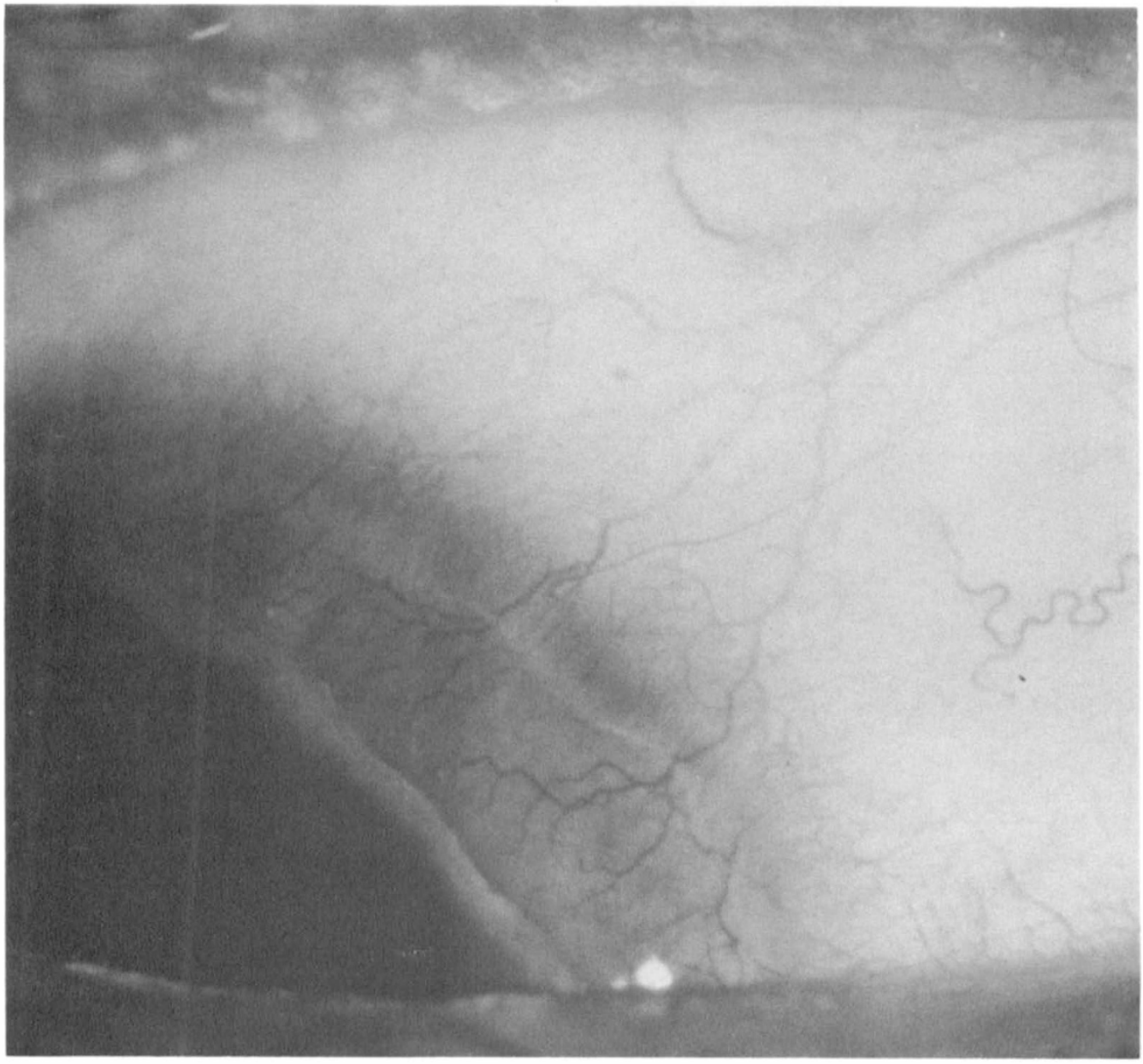

Fig. 10. Early Mooren's ulceration. There is no limbal inflammation in spite of very active destructive corneal changes. The edge of the ulcer is undermined and infiltrated and the cornea beyond thickened. The limbal arcade is disrupted and conjunctival vessels pass to the edge of the ulcer. They are not joined together but arborise.

whether the disease is active and progressive or is in a quiescent phase. The earliest indication of disease is a disruption of the normal capillary architecture. During this active phase of the disease the new vessel sprouts are straight and leak at their tips, but as soon as the disease becomes quiescent they join up with adjacent vessels to form a mature arcade. Leakage from the tips ceases at this stage.

\section{Discussion}

If those conditions which are caused directly by tear film abnormalities and destructive changes caused by micro-organisms or the immune response to them are excluded, there remains a group of conditions which are characterised by two features. The first is that the destructive changes commence within $2 \mathrm{mms}$ of the limbus and the second is that they are all accompanied by a degree of vasoocclusion of the adjacent vascular supply at the limbus.

Although there does not appear to be a characteristic vascular change which separates one condition from another, anterior segment flourescein angiography records changes in each of these conditions, all of which involve vaso-obliteration of part of the vascular tree, either in the limbal arcades, in 


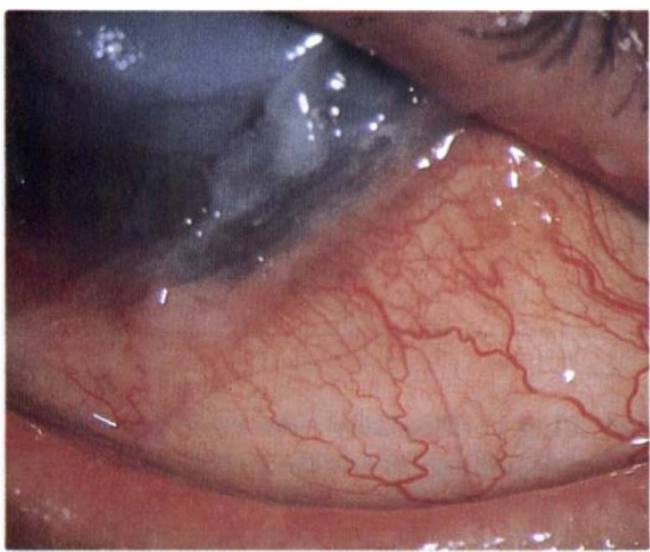

Fig. 11a. Active Moorens ulceration. The disease involves the whole cornea. The periphery of the cornea is very thin but intact. The centre of the cornea is opaque and oedematous. The abnormal mucus is a characteristic finding in all patients with peripheral corneal ulceration in its active phase.

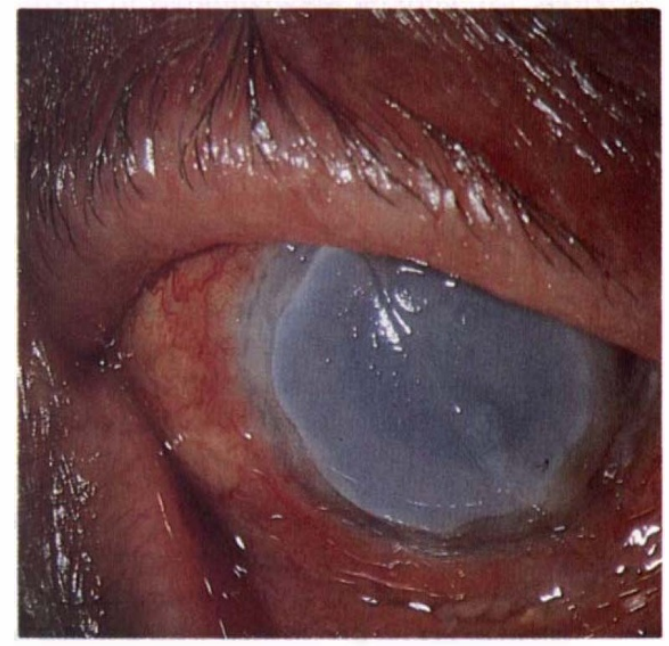

Fig. 11b. The other eye of the patient in 11a showing an earlier stage of the disease. The central cornea is cloudy but not totally opaque. The ulceration is circumferential and an infiltrate is present at the advancing edge of the ulcer.

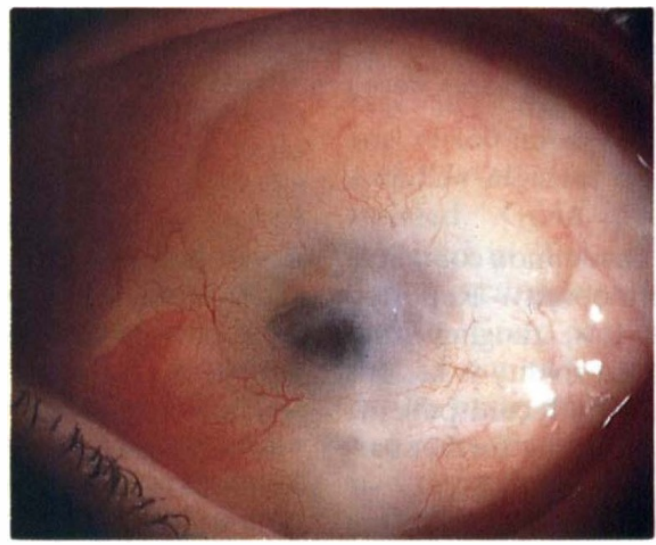

Fig. 12. Spontaneous resolution in Mooren's ulceration. The corneal stroma has been absorbed leaving Descemets membrane with a thin covering of epithelium and scar tissue.

the episcleral meshworks, or in the larger superficial and deep vascular networks. ${ }^{2}$ Although it is not absolute, the larger the area of vaso-occlusion, the greater degree of corneal or scleral destruction, thus in 'contact lens' cornea the vaso-occlusive changes largely affect the limbal arcades and the capillary networks adjacent to them. In conditions where there are large areas of tissue loss, for example Mooren's ulceration and acute stro- mal keratitis the vaso-occlusion is widespread involving both large and small vessels.

\section{References}

${ }^{1}$ Meyer P. Limbal vascular changes in ocular systemic inflammatory diseases. Brit J Ophthalmol 1989 (Submitted for Publication).

${ }^{2}$ Watson PG and Bovey E: Anterior segment fluorescein angiography in the diagnosis of scleral inflammation. Ophthalmology 1985, 1: 1-11. 